\title{
Modelo de lote económico de pedido EOQ en el inventario de partes de servicio automotriz
}

\author{
Economic order model EOQ in the inventory of automotive service parts
}

Arturo Contreras-Juárez ${ }^{a}$, Marisol Escalante-Herrera ${ }^{b}$, Ismael Cortes-Maldonado ${ }^{c}$, Francisco

Baños-Islas d

\begin{abstract}
:
The supply chain is integrated into a network of logistics operations by suppliers, manufacturers, intermediate distributors and finally consumers. Where inventories play the most important role in meeting the exact times and quantities of customer demand, this way setting policies at inventory levels minimizes the risk of late deliveries and helps to reduce total logistics costs. Assuming that inventory management is a fundamental part of business strategic and operational planning. This article therefore addresses the use of the economic order model (EOQ) in an automotive parts spare parts warehouse, establishing inventory policies for spare parts demand in the maintenance area, taking into account the variability of demand and supply times in the application of the model, the optimal quantities to be ordered from the parts of service with respect to the duration of the order cycle are established, supporting the decisions to be taken in the inventory levels of the parts of service, minimizing the logistical costs and improving the quality of the customer service.
\end{abstract}

\section{Keywords:}

Inventory policy, automotive service parts, logistics costs.

\section{Resumen:}

La cadena de suministro está integrada dentro de una red de operaciones logísticas de proveedores, fabricas, distribuidores intermedios y finalmente los consumidores. Donde los inventarios juegan el rol más importante por cumplir con los tiempos y las cantidades exactas de la demanda del cliente, de esta forma el establecer las políticas en los niveles de inventario minimiza el riesgo de entregas a destiempo y ayuda a reducir los costos logísticos totales. Asumiendo que la administración de los inventarios es una parte fundamental de la planeación estratégica y operativa de las empresas. El presente artículo aborda el uso del Modelo de Lote Económico de Pedido (Economic Order Quantity - EOQ) en un almacén de refacciones de partes de servicio automotriz, estableciendo políticas de inventarios para la demanda de refacciones en el área de mantenimiento, tomando en cuenta la variabilidad de la demanda y los tiempos de suministro en la aplicación del modelo, quedando establecidas las cantidades optimas a ordenar de las partes de servicio con respecto al tiempo de duración del ciclo de pedido, brindando soporte a las decisiones que han de tomarse en los niveles de inventario de las refacciones de partes de servicio, minimizando los costos logísticos y mejorando la calidad del servicio al cliente.

\section{Palabras Clave:}

Política de inventario, partes de servicio automotriz, costos logísticos

\footnotetext{
a Autor de Correspondencia, Universidad Politécnica de Tlaxcala Región Poniente, https:// orcid.org/0000-0002-7192-3596, Email: arturoconteras@uptlaxponientes.edu.mx.

b Universidad Politécnica de Tlaxcala Región Poniente, Email: marisolescalante42@ gmail.com.

c Universidad Politécnica de Tlaxcala Región Poniente, Email: ismaelcortes@uptlaxponiente.edu.mx.

duniversidad Tecnológica de Tlaxcala. Tlaxcala, Email: banosislas@uttlaxcala.edu.mx.
} 


\section{Introducción}

Los intercambios de bienes y servicios en todos los niveles y modalidades en los rincones más apartados del planeta son una realidad, gracias a la globalización. Por lo que la compra y venta de artículos en la cantidad, calidad y tiempo de entrega deseado son un requisito que le permite a las empresas acumular los inventarios suficientes para satisfacer las demandas de sus clientes. 1

El costo anual de almacenamiento puede oscilar entre el $14 \%$ y el $36 \%$ del valor promedio de los productos almacenados. Los administradores del inventario deben determinar los límites, la magnitud y la composición de cada inventario antes de tomar una decisión en términos de los objetivos de la organización. Los almacenes y centros de distribución ocupan una posición muy importante en la recuperación de productos para satisfacer las órdenes de los clientes.

Dentro de las operaciones de un almacén, la preparación de pedidos, se encarga de recuperar los ítems requeridos por las órdenes de los clientes, los cuales se encuentran en distintas posiciones de almacenamiento. Esto hace que la operación sea más intensiva en mano de obra dentro de los almacenes manuales, generando el $60 \%$ y el $70 \%$ del costo operativo total de un almacén. La gestión de sistemas de inventarios constituye una de las funciones más complejas de las organizaciones, ya que implica mantener existencias para protegerse contra incertidumbres al menor costo. Una política de inventario debe dar respuesta a las preguntas de cada cuando debe revisarse el inventario, cuando y cuanto ordenar, bien sean ítems de demanda independiente $\mathrm{o}$ dependiente. Sin embargo, la metodología de estimación de políticas para darle respuesta a estas preguntas puede variar significativamente debido a dos aspectos: el tipo de producto (terminado o materia prima) y el ambiente de producción. 2-6

Los inventarios en proceso y los inventarios de producto final constituyen un aspecto de gran importancia para la organización y son un punto de partida para la toma de decisiones estratégicas de la empresa. Toda empresa, sea de producción, comercialización $o$ de servicios requiere de aprovisionamiento de productos para llevar a cabo sus actividades de producción y/o venta y por consiguiente la existencia de inventarios. 7,8
Todo pedido al proveedor implica realizar un proceso de solicitud de abastecimiento y almacenamiento de mercancía, por lo tanto, se generan dos costos asociados a la orden: el costo de pedir y el costo de mantener el inventario, cuya suma es en esencia, el costo total del manejo del inventario.

El tamaño económico de lote (EOQ) es sencillamente la cantidad a pedir al proveedor, de un determinado artículo, donde la suma de los costos de ordenar y de mantener inventario es óptimo. Este modelo es fundamental para todos los modelos de inventario. Describe el equilibrio entre los costos de pedido y los costos de mantener el inventario, y es la base para el análisis de sistemas más complicados.

\section{Materiales y métodos}

Si bien es cierto que algunas empresas utilizan sistemas informáticos de alta especialidad para el control y administración de sus almacenes, no quiere decir que no se puedan hacer mejoras o diseñar modelos cuantitativos para apoyar al control de los inventarios y sus costes.

Para identificar y tomar la decisión del modelo EOQ aplicado se determina el coeficiente de variabilidad (CV). Si el coeficiente determinado es menor a 0,20 el cual es representado en porcentaje $(20 \%)$ se implementa un modelo determinístico, de lo contrario la demanda es muy inestable y corresponde la aplicación de un modelo probabilístico. 9

Cuando se desea hacer referencia a la relación entre el tamaño de la media y la variabilidad de la variable, se utiliza el coeficiente de variabilidad (CV). Su fórmula expresa la desviación estándar como porcentaje de la media aritmética, mostrando una mejor interpretación porcentual del grado de variabilidad que la desviación típica o estándar. A mayor valor del coeficiente de variación mayor heterogeneidad de los valores de la variable; y a menor CV, mayor homogeneidad en los valores de la variable, por lo que se recomienda que el CV no sea mayor al $20 \%$.

Se calcula:

$$
\mathrm{C} v=\sigma / \dot{\mathrm{x}}
$$

donde:

$\sigma$. es la desviación típica, y

$\dot{x}$ es la media. 
Se puede dar en porcentaje calculando:

Si el coeficiente es menor a $0.20(20 \%)$ se puede usar un modelo determinístico, de lo contrario la demanda es muy inestable y no se podrá aplicar este modelo.

\section{Modelo determinístico}

Cálculo del Modelo Determinístico de Lote Económico de Pedido (EOQ). 10

Cantidad óptima a ordenar:

$q^{*}=\sqrt{\frac{2 K d}{h}}$

Costo total logístico de inventario:

$$
\mu(q)=\frac{K d}{q}+c d+\frac{h q}{2}
$$

Duración del ciclo de pedido:

$$
n=\left(\frac{d}{q^{*}}\right)
$$

Número de órdenes anuales:

$$
T^{*}=\frac{q^{*}}{d}
$$

\section{Dónde:}

d: Demanda determinística

$q^{*}$ : Tamaño óptimo de cada pedido

q: Tamaño de cada pedido

$\mathrm{K}$ : Costo de ordenar

h: Costo de sostener inventario

c: Costo de compra

Para el desarrollo y aplicación del modelo se asignó un catálogo de 20 números de partes de servicio proporcionados por el jefe de almacén de una empresa de mantenimiento vehicular, ya que son refacciones que de acuerdo a la experiencia del jefe de almacén son de alta rotación y en algunos casos han presentado desabasto ya sea por una mala planeación del departamento de compras o por hacer requisiciones con un número de piezas sin fundamento o por falta de existencias en el almacén del proveedor.
La Tabla 1. Presenta el catálogo de 20 refacciones de estudio, la unidad de medida como son solicitados a los proveedores y despachados a los técnicos en el área de mantenimiento.

\begin{tabular}{clll}
\hline No. & Refacciones & Medida & Proveedor \\
\hline 1 & Aceite 20W/40 & Litro & Mobil S.A. de C.V. \\
2 & Balata & Juego (2) & Villacero S.A de C.V \\
3 & Llantas & Pieza & Llantera Garay S.A. de C.V. \\
4 & Filtro de aceite & Pieza & Fleetguard \\
5 & Filtro de agua & Pieza & Fleetguard \\
6 & Filtro de combustible & Pieza & Fleetguard \\
7 & Filtro separador de agua & Pieza & Fleetguard \\
8 & Filtro de aire & Pieza & Fleetguard \\
9 & Anticongelante & Litro & Texaco \\
10 & Matraca & Pieza & Didiasa S.A. de C.V. \\
11 & Válvula niveladora de suspensión & Pieza & Mangueras y conexiones de Mexico S.A. de \\
12 & Manguera de aire de 1/2 & Metro & Mangueras y conexiones de Mexico S.A. de \\
13 & Conexión rápida de aire de 1/2 & Pieza & Mangueras y conexiones de Mexico S.A. de \\
14 & Plumas limpia brisas & Juego (2) & Didiasa S.A. de C.V. \\
15 & Bolsa de aire & Pieza & Didiasa S.A. de C.V. \\
16 & Bujes de Sta rueda & Juego (2) & Didiasa S.A. de C.V. \\
17 & Grasa estándar & Kilo & Didiasa S.A. de C.V. \\
18 & Válvula de aire para llanta & Pieza & Mangueras y conexiones de Mexico S.A. de \\
19 & Soldadura E6013 & Kilo & INFRA \\
20 & Cepelines & Pieza & APYMSA \\
\hline
\end{tabular}

Tabla 1. Catálogo de refacciones para proceso de estudio.

(Elaboración Propia)

La tabla 2 presenta el catálogo de las refacciones en estudio, con el promedio diario de solicitud para su uso en el taller mecánico de la empresa. Donde se observa que la refacción con mayor demanda de uso es el aceite con 117 litros al día, seguida de la grasa estándar con 35 kilos por día y la menos usada son los filtros con 2 unidades por día.

\begin{tabular}{clc}
\hline No. & Refacciones & Promedio de uso por día \\
\hline 1 & Aceite 20W/40 & 117 \\
2 & Balata & 12 \\
3 & Llantas & 7 \\
4 & Filtro de aceite & 2 \\
5 & Filtro de agua & 2 \\
6 & Filtro de combustible & 2 \\
7 & Filtro separador de agua & 2 \\
8 & Filtro de aire & 2 \\
9 & Anticongelante & 30 \\
10 & Matraca & 7 \\
11 & Válvula niveladora de suspensión & 9 \\
12 & Manguera de aire de $1 / 2$ & 11 \\
13 & Conexión rápida de aire de 1/2 & 15 \\
14 & Plumas limpia brisas & 5 \\
15 & Bolsa de aire & 4 \\
16 & Bujes de 5ta rueda & 6 \\
17 & Grasa estándar & 35 \\
18 & Válvula de aire para llanta & 6 \\
19 & Soldadura E6013 & 12 \\
20 & Cepelines & 10 \\
\hline
\end{tabular}

Tabla 2. Promedio de solicitud de refacciones por día para su uso.

(Elaboración Propia)

La grafica 1 presenta las 20 refacciones y el porcentaje de la demanda promedio por día en el taller mecánico esta demanda es por los mantenimientos preventivos programados o por los mantenimientos correctivos. 


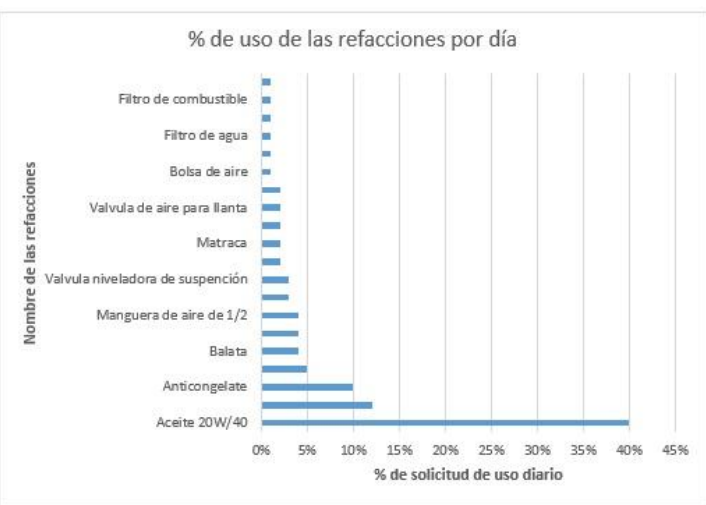

Gráfica 1. Porcentaje de representación de uso de las refacciones.

(Elaboración Propia)

El estudio realizado a la empresa muestra que no cuenta con la aplicación de metodologías cuantitativas para el control de la logística de inventarios, el cual afecta directamente en el servicio de mantenimiento externando quejas en un promedio de $30 \%$ en incumplimiento de fechas y horarios de entrega de las refacciones y un $20 \%$ de inconformidades por entregas incompletas en el taller mecánico.

El retraso por incumplir con los requerimientos en el taller, genera un $35 \%$ de re trabajos, afectando en salidas tardías de acuerdo a los tiempos establecidos para la entrega de las unidades vehiculares, con un $40 \%$ de tiempo excedente y genera un $36 \%$ de tiempo extra en mano de obra en el área de mantenimiento. Se estima un incremento del $35 \%$ de gastos administrativos en (Llamadas telefónicas, papelería, salarios administrativos, refacturaciones, generación de nuevas órdenes de pedido, servicio al cliente y combustible).

La tabla 3 presenta una clasificación $\mathrm{ABC}$, a fin de identificar las refacciones con mayor demanda en el taller, donde para la aplicación de los modelos se utilizan las refacciones que se ubican en la clasificación "A" por el grado de importancia que estas representan.

\begin{tabular}{|c|l|c|c|c|c|}
\hline No. & \multicolumn{1}{|c|}{ Refacciones } & $\begin{array}{c}\text { Promedio } \\
\text { de uso por } \\
\text { dia }\end{array}$ & $\begin{array}{c}\% \text { de } \\
\text { representación }\end{array}$ & $\begin{array}{c}\% \\
\text { acumulado }\end{array}$ & $\begin{array}{c}\text { Clasificación } \\
\text { ABC }\end{array}$ \\
\hline 1 & Aceite 20W/40 & 117 & $40 \%$ & $40 \%$ & \\
2 & Grasa estándar & 35 & $12 \%$ & $51 \%$ & \\
3 & Anticongelante & 30 & $10 \%$ & $61 \%$ & \\
4 & Conexión rápida de aire de 1/2 & 15 & $5 \%$ & $67 \%$ & A \\
5 & Balata & 12 & $4 \%$ & $71 \%$ & \\
6 & Soldadura E6013 & 12 & $4 \%$ & $75 \%$ & \\
7 & Manguera de aire de 1/2 & 11 & $4 \%$ & $78 \%$ & \\
\hline 8 & Cepelines & 10 & $3 \%$ & $82 \%$ & \\
9 & Válvula niveladora de suspension & 9 & $3 \%$ & $85 \%$ & \\
10 & Llantas & 7 & $2 \%$ & $87 \%$ & \\
11 & Matraca & 7 & $2 \%$ & $90 \%$ & B \\
12 & Bujes de Sta rueda & 6 & $2 \%$ & $92 \%$ & \\
13 & Válvula de aire para llanta & 6 & $2 \%$ & $94 \%$ & \\
14 & Plumas limpia brisas & 5 & $2 \%$ & $95 \%$ & \\
\hline 15 & Bolsa de aire & 4 & $1 \%$ & $97 \%$ & \\
16 & Filtro de aceite & 2 & $1 \%$ & $97 \%$ & \\
17 & Filtro de agua & 2 & $1 \%$ & $98 \%$ & C \\
18 & Filtro de aire & 2 & $1 \%$ & $99 \%$ & \\
19 & Filtro de combustible & 2 & $1 \%$ & $99 \%$ & \\
20 & Filtro separador de agua & 2 & $1 \%$ & $100 \%$ & \\
\hline
\end{tabular}

Tabla 3. Clasificación $A B C$ por demanda de las refacciones en estudio.

(Elaboración Propia)
La Tabla 4. Presenta el modelo que aplica por refacción de acuerdo al coeficiente de variabilidad obtenido de la aplicación de la fórmula 1, descrita en la sección de metodología.

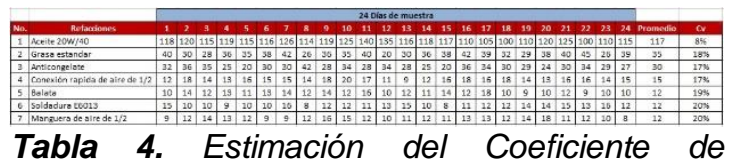

Variabilidad para asegurar el modelo propuesto EOQ.

(Elaboración Propia)

El ciclo de aplicación del modelo EOQ se representa gráficamente en la Figura 3 la cual interpreta el tiempo que ha de tardar cada pedido, así como el punto de reorden para cada pedido y los niveles de stock requeridos de acuerdo al desarrollo del modelo.

La tabla 5 presenta los datos cuantitativos de las variables requeridas para la aplicación del modelo EOQ, como son el costo por pedido, la demanda anual, el costo por sostener 1 unidad en el inventarío el costo por comprar una unidad, el tiempo de entrega de las refacciones por parte de los proveedores y el costo por unidad de cada una de las refacciones de la clasificación $A$.

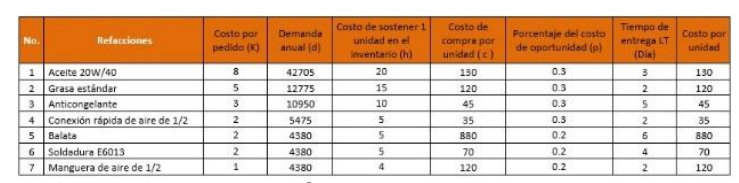

Tabla 5. Asignación de datos en las variables del modelo EOQ. (Elaboración Propia)

De los datos establecidos en la tabla 4 para la aplicación del Modelo de Lote Económico se estima la cantidad optima a ordenar en cada uno de los pedidos de las refacciones, la duración del ciclo de pedido en días que tendrá cada proveedor para la entrega de refacciones en el almacén, se estima el número de órdenes de requisición de refacciones que se deberá de hacer en un periodo de un año, así como el punto de reorden el cual establece el stock mínimo en el inventario para hacer la requisición de las refacciones, de esta manera se estiman los costos logísticos del inventario total para el periodo anual.

\section{Resultados}

La tabla 6 presenta los resultados obtenidos de la aplicación del Modelo de Lote Económico $E O Q$. Donde se estimó la cantidad óptima a ordenar de cada una de las refacciones donde se visualiza que la de mayor cantidad a solicitar es el Aceite 20W/40 y la mínima es la de Manguera de aire de $1 / 2$. Donde en la duración del ciclo de pedido en días la que tardará más en llegar será 
la balata y la soldadura E6013 con un estimado de 5 días. Por otra parte, la refacción que requiere el mayor número de ordenes anuales es el aceite con 231 órdenes de requisición generando así los costos logísticos totales anualizados como es el caso del anticongelante que será de $\$ 493,560.55$.

\section{Conclusiones}

El establecer políticas de inventario a través de los modelos de lote económico de pedido (Economic Order Quantity -EOQ) orienta a los administradores de almacén a elaborar una planeación logística de los inventarios, identificando con mayor certidumbre los volúmenes de pedido a fin de garantizar las demandas de los clientes sin generar excedente, garantizando el nivel de servicio máximo.

Con los resultados obtenidos se establece que la aplicación de métodos cuantitativos para generar políticas de inventario genera resultados positivos en beneficio de las organizaciones y que estas no están limitadas a un sector comercial, y que los almacenes de refacciones de partes de servicio requieren de estas metodologías en apoyo a minimizar sus costos logísticos de inventario para el aumento de su rentabilidad

\section{Referencias}

[1] Bustos, C. y Chacón. G. Modelos determinísticos de inventarios para demanda independiente. Un estudio en Venezuela. Contaduría y administración. 2010; 57 (3): 239-258

[2] Estrada, S., Restrepo, L., y Ballesteros, P. (2010) Análisis de los costos logísticos en la administración de la cadena de suministro. Universidad Tecnológica de Pereira. Vol. (16). No. 45. pp. 272-277. Colombia.

[3] Fogarty, D., Blackstone, J. y Hoffmann, T. (2016) Administración de la producción e inventarios 2da Ed. Grupo Editorial Patria.

[4] Bustillo, M., Menéndez, B., Pardo, E., y Duarte, A. (2015). An Algorithm for Batching, Sequencing and Picking operations in a Warehouse. International Conference on Industrial Engineering and Systems Management, Sevilla, España. DOI:10.1109/IESM.2015.7380254

[5] Chen, T., Cheng, C., y Chen, Y., y Chan, L. (2015). An Efficient Hybrid Algorithm for Integrated Order Batching, Sequencing and Routing Problem. International Journal of Production Economics. Vol. (159). 158-167. https://doi.org/10.1016/j.ijpe.2014.09.029

[6] Gutiérrez, V. y Vidal, C. (2008). Modelos de Gestión de Inventarios en Cadenas de Abastecimiento: Revisión de la Literatura. Rev. Fac. Ing. Univ. Antioquia No. (43). pp. 134-149. http://www.scielo.org.co/pdf/rfiua/n43/n43a12.pdf

[7] Causado, E. (2015). Modelo de inventarios para control económico de pedidos en empresa comercializadora de alimentos. Revista Ingenierías Universidad de Medellín, Vol. (14). No. 27. pp. 163-178. ISSN:1692 - 3324. https://doi.org/10.22395/rium.v14n27a10

[8] Peña, O y Silva, R. (2016). Factores incidentes sobre la gestión de sistemas de inventario en organizaciones venezolanas. Revista de Estudios Interdisciplinarios en Ciencias Sociales. Vol. 18 (2). pp. 187 - 207. ISSN 1317-0570. http://www.redalyc.org/pdf/993/99345727003.pdf

[9] Palacios, L., Flores L., Álvarez, R., Contreras, A., Sánchez, D., y Martínez, J. (2015). Inventory management through push y pull and vendor management inventory - case of study: RI SA products. DYNA Management. Vol.3 pp. 1-8. DOI: http://dx.doi.org/10.6036/MN7565

[10] Taha, H. (2012). Investigación de operaciones. Novena edición. Pearson educación, México. ISBN: 978-607-32-07966. 